\title{
Simultaneous mapping of temporally-resolved blood flow velocity and oxygenation in femoral artery and vein during reactive hyperemia
}

\author{
Michael C Langham and Felix W Wehrli
}

\begin{abstract}
Background: Post-occlusive hyperemia is often used as a paradigm to evaluate vascular reactivity, for example by measuring post-ischemic flow-mediated dilation, arterial blood flow or temporally resolved venous blood oxygenation $\left(\mathrm{HbO}_{2}\right)$. Here we demonstrate the feasibility of a simultaneous measurement of blood flow and $\mathrm{HbO}_{2}$ in the femoral circulation as part of a single procedure.

Methods: A multi-echo GRE pulse sequence was designed and implemented to collect velocity-encoded projections in addition to full-image echoes for field mapping as a means to quantify intravascular magnetic susceptibility. The method's feasibility was evaluated at 3T in a small pilot study involving two groups of healthy subjects (mean ages $26 \pm 1.6$ and $59 \pm 7.3$ years, $N=7$ and 5, respectively) in terms of six parameters characterizing the time-course of reactive hyperemia and their sensitivity to differentiate age effects. The reproducibility was assessed on two of the seven young healthy subjects with three repeated measurements.

Results: The physiological parameters agree with those obtained with current methods that quantify either velocity or $\mathrm{HbO}_{2}$ alone. Of the six measures of vascular reactivity, one from each group was significantly different in the two subject groups $(p<0.05)$ even though the study was not powered to detect differences. The mean coefficient of variation (CV) from two subjects undergoing repeat scans were approximately $8 \%$ for the oximetric and the arterial velocimetric parameters in the femoral vein and artery, respectively, considerably below intersubject CVs (20 and 35\%, for the young and older subject groups, respectively).

Conclusion: The proposed method is able quantify multiple parameters that may lead to more detailed assessment of peripheral vascular reactivity in a single cuff paradigm rather than in separate procedures as required previously, thereby improving measurement efficiency and patient comfort.
\end{abstract}

\section{Background}

In the United States, cardiovascular diseases (CVD) account for more deaths than cancers, chronic obstructive pulmonary diseases and accidents combined [1]. The burden of CVD in the future is expected to grow due to the aging population, prevalence of obesity $[2,3]$ and unhealthy lifestyle choices that include smoking [4], lack of physical activity [5] and poor nutrition [6,7]; all factors predisposing subjects to develop systemic atherosclerosis that can remain silent for decades. The earliest stage of pathogenesis of atherosclerosis, the most

\footnotetext{
*Correspondence: wehrli@mail.med.upenn.edu

Laboratory for Structural NMR Imaging, Department of Radiology, University of Pennsylvania Medical Center, Philadelphia, PA, USA
}

common cause of CVD, is endothelial dysfunction (EDF), which can manifest as impaired reactivity in the peripheral vascular bed (e.g. brachial or femoral arteries) due to the suppression of nitric oxide (NO) [8] synthesis. In normal vascular function greater demand for blood flow in response to a physiological challenge is accommodated initially via reduction of microvascular resistance. Subsequently, increase in shear stress to the vessel wall promotes NO release resulting in vasodilation (referred to as flow-mediated dilation (FMD)) of the conduit artery [9]. Thus, micro- and macrovascular function can be assessed during reactive hyperemia.

Among non-invasive imaging modalities used to quantify reactive hyperemia in peripheral arteries are nearinfrared spectroscopy (NIRS) [10-13], single photon

\section{C) Biomed Central}


emission computed tomography [14], cardiovascular magnetic resonance (CMR) [15-18], and ultrasound [19]. NIRS and ultrasound (US) are most commonly used due to their portability. NIRS is relatively inexpensive, has excellent temporal resolution and is less prone to artifacts from subject motion. It also allows simultaneous monitoring of relative changes in tissue blood flow and oxygenation [12]. However, the method's spatial resolution is limited and can only provide microvascular function in relatively superficial tissues. In addition, the tissue response to light depends on skin color, body fat and muscle layers, leading to large intersubject variations [20]. US measurements of FMD in the brachial artery have generated substantial interest in clinical research since its introduction [21]. Numerous studies have since demonstrated the prognostic value of brachial artery FMD [22,23]. However, the methodology suffers from substantial inter- and intra-observer variability $[9,24]$ in that probe placement or US settings such as dynamic range, gain and probe distance can significantly affect diameter measurements [25]. These sources of error are of particular concern since the average relative change of FMD is on the order of $5 \%$ only [26].

CMR has also demonstrated its potential for evaluating vascular reactivity. CMR flow velocimetry can be performed with high spatial and temporal resolution as demonstrated in a recent study in which post-occlusion hyperemia in the femoral artery was evaluated in PAD patients and healthy subjects $1.5 \mathrm{~T}$ [16]. The implementation of the method requires spatially-selective rf pulses which are not widely available and migration to systems with higher field strength may be challenging due to increased field inhomogeneity. Alternatively, microvascular function can be assessed by quantifying post-occlusive perfusion with arterial spin labeling (ASL) [17] in calf muscle. The ASLapproach is model-dependent and the temporal resolution is limited.

All of the above methods only target one aspect of the vascular reactivity, e.g. hemodynamics, tissue oxygenation, or FMD, as possible indicators of endothelial dysfunction. We posit that quantification of several physiological parameters may lead to a more sensitive assessment for detecting early stage of EDF. As a step toward an integrated CMR protocol to detect early stages of EDF and to extend a MR procedure developed previously based on using oxygen saturation as a dynamic tracer [18], we describe an interleaved multiecho gradient-recalled (GRE) sequence that is able of temporally resolve venous blood oxygen saturation and flow velocity simultaneously during hyperemia. The feasibility and reproducibility of the proposed method is demonstrated in seven young and five older healthy subjects.

\section{Methods}

Integration of MR susceptometry and projection-based velocity quantification

MR susceptometry relies on the quantification of the $\mathrm{HbO}_{2}$-dependent susceptibility difference between intravascular blood and surrounding tissue $[27,28]$. For the remainder of the manuscript $\mathrm{HbO}_{2}$ is defined as the fractional oxygen saturation expressed in percent. The relative susceptibility difference can be derived from a phase difference image between two gradient echoes differing in echo time. Velocity is computed from a phase difference image between interleaved GREs differing in velocity-encoding. The basic idea of integrating MR susceptometry and velocity quantification is to interleave multi-echo GREs with different velocity encoding [27]. However, in order to achieve adequate temporal resolution to map the time-course of hyperemia, the velocity will be quantified with projections [29] while full 2D echoes will be acquired for $\mathrm{HbO}_{2}$ quantification.

\section{Pulse Sequence}

The pulse sequence for temporally-resolved measurement of venous oxygen saturation and flow velocity is shown in Figure 1a. It consists of a fat-suppressed multi-echo GRE pulse sequence for collecting velocityencoded projections sampled at TE1 (i.e. in the absence of phase-encoding). The same excitation generates two additional echoes (TE2 and TE4), which are phaseencoded, thereby yielding a full image for field mapping. The velocity encoding is toggled after each TR by separating the flow-compensating gradient lobes appropriately (dashed box Figure 1a); the maximum temporal resolution is $2 \times \mathrm{TR}$. Thus, while the pulse sequence collects velocity-encoded projections at TE1 the phaseencoding gradient is incremented for the remaining echoes. In this manner, 64 pairs of velocity-encoded projections can be collected while a single $2 \mathrm{D}$ field map is obtained from TE2 and TE4. The sequence in Figure $1 \mathrm{~b}$ collects a 2D reference image at TE1 for removing background tissue signal from the velocity-encoded projections, as explained in more detail below. In addition, data are acquired at TE2 and TE4 for 2D field mapping. In this manner, uninterrupted measurement of $\mathrm{HbO}_{2}$ is ensured. We note that TE2 and TE4 have the same first moment in all three directions so that flow-induced phase accumulation is minimized for the field maps.

\section{Subjects}

Seven healthy young (YH, $26 \pm 1.6$ yrs) and five older healthy $(\mathrm{OH}, 59 \pm 7.3 \mathrm{yrs})$ subjects without prior history of cardiovascular disease were recruited to evaluate the performance of the interleaved pulse sequence in terms of reproducibility and the potential to detect expected age-related differences between the two groups with 


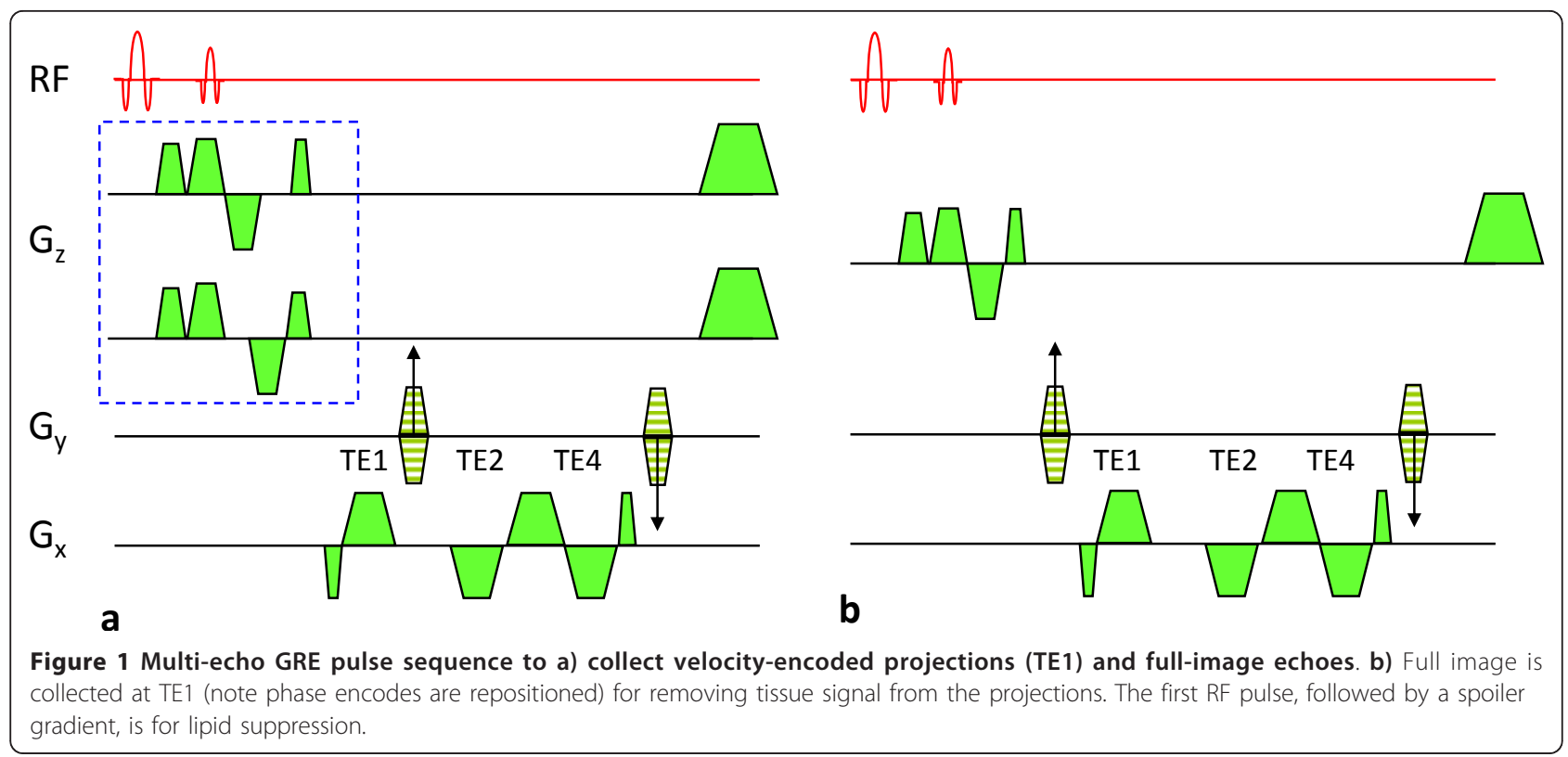

parameters of vascular reactivity. To ascertain that all subjects were free of peripheral arterial disease the ankle-brachial index (ABI) was determined. All subjects evaluated had ABI > 0.9. Written informed consent was obtained prior to all examinations following an institutional review board-approved protocol.

\section{Cuff paradigm}

Ischemia was induced by inflating a blood pressure cuff (Aspen Labs A.T.S 1500 Tourniquet System) to 75 $\mathrm{mmHg}$ above the systolic pressure in the upper thigh. The location was chosen so as to minimize perturbation of the imaging region (approximately $10 \mathrm{~cm}$ superior to the knee) during cuff inflation or deflation. On ten subjects $(5 \mathrm{YH}$ and $5 \mathrm{OH})$, the cuff paradigm consisting of 2 mins baseline, 5 mins occlusion and 6 mins recovery was performed to monitor post-ischemic vascular reactivity.

\section{Reproducibility}

Two YH subjects (not taken from the pool of $5 \mathrm{YH}$ ) participated only in the reproducibility study where an abbreviated cuff paradigm (3 mins occlusion and 2 mins recovery) was used in order to allow for three repeated measurements in a single session to assess reproducibility. After each scan the subject was removed from the scanner and allowed to rest for 15 mins to ensure full recovery before repeating cuff occlusion.

\section{CMR Protocol}

All imaging was performed on a $3 \mathrm{~T}$ Siemens Trio. Phased-array eight-channel knee coil (Invivo Inc., Pewaukee, WI) was used to acquire axial images of femoral vessels. From the scout images an appropriate readout direction was chosen to avoid overlap between the femoral artery and vein along the projection direction. For the data acquisition the following imaging parameters were used: TE/TR $=5.0 / 39.1 \mathrm{~ms}$, flip angle $=13^{\circ}$, Bandwidth $=520 \mathrm{~Hz} /$ pix, voxel size $=1 \times 1 \times 5$ $\mathrm{mm}^{3}$, matrix size $=128 \times 128$. In addition to acquiring velocity-encoded projections taken with equal magnitude but opposite-sign first moments, flow-compensated projections (not shown in Figure 1) were also acquired repeatedly to ensure acquisition of unaliased phase data. Thus, three projections offer the flexibility of achieving either VENC of 125 or $250 \mathrm{~cm} / \mathrm{s}$, as needed, i.e. by computing the phase difference between acquisitions taken with opposite polarity flow-encoding gradients or between zero and non-zero first-moment encoded projections. The pulse sequence of Figure 1a was launched $10 \mathrm{~s}$ prior to and was run for $60 \mathrm{~s}$ after the cuff release. For the remaining recovery period only $\mathrm{HbO}_{2}$ was monitored with the flow-compensated pulse sequence (Figure 1b). The pulse sequence achieves temporal resolutions of $5 \mathrm{~s}$ and $117 \mathrm{~ms}$ for dynamic oximetry and velocity quantification, respectively.

\section{Data Analysis \\ $\mathrm{HbO}_{2}$ quantification}

The raw k-space data were processed off-line. A phase difference image was computed with the images of equal polarity echoes, e.g. TE2 and TE4. The effects of low spatial-frequency modulations of static magnetic field were minimized by fitting the data, after appropriate weighting and masking, to a second-order polynomial [30]. The hemoglobin oxygen saturation, $\mathrm{HbO}_{2}$, in 
the femoral vein was
$\mathrm{HbO}_{2}=\left[1-\frac{6|\Delta \phi| / \Delta T E}{\gamma \Delta \chi_{d_{o}} H c t \cdot B_{o}\left(3 \cos ^{2} \theta-1\right)}\right][1]$.

where $\Delta \varphi$ is the average intravascular phase relative to surrounding tissue, $\triangle T E=T E 4-T E 2$, is the inter-echo, $\gamma$ is the gyromagnetic ratio of water protons, $\Delta \chi_{d o}=4 \pi$ $\times(0.27 \pm 0.02) \mathrm{ppm}[31]$ is the susceptibility difference between fully deoxygenated and fully oxygenated erythrocytes in SI units, $\theta$ is the vessel tilt angle relative to the main field $B_{o}$ and $H c t$ (hematocrit) is the volume fraction of erythrocytes in packed blood.

The time-course of $\mathrm{HbO}_{2}$ characterize vascular reactivity with three parameters: washout time, upslope and overshoot, as described previously [18]. The washout time refers to the elapsed time to observe oxygendepleted capillary blood at the measurement location after cuff deflation $(t=0)$. Resaturation occurs at a mean rate termed upslope and the transient increase in blood flow rate leads to an overshoot, an above-baseline $\mathrm{HbO}_{2}$ level.

\section{Blood velocity quantification}

In order to isolate the vascular signal from static tissue a projection derived from the reference image is subtracted from the velocity-encoded projections as described previously [29]. In brief, the vessels of interest are masked out manually from the complex reference image and then Fourier transformed to k-space. The projection, which contains tissue signal only, is then subtracted from the velocity-encoded projections, thus isolating blood signal. The subtraction is performed in the complex domain to retain the phase information. The phase differences between velocity-encoded projections are converted to velocity using the relation between phase $\varphi$ and velocity $v$, as $v=\varphi \cdot V E N C / \pi$. The spatially averaged velocities of the vessels of interest are derived by averaging the velocity along the readout direction within the vessel boundaries. Thus, at each temporal point, the average velocity represents the velocity averaged over the lumen.

The following parameters are derived from the timeresolved blood flow velocity: time required to reach peak average velocity (time-to-peak (TTP)), the duration of forward flow $\left(T_{f f}\right)$ and maximum change in the flow rate relative to baseline $\left(\Delta Q_{\max }\right)$, which we define as the ratio, $v_{\text {peak, avg }} \nu_{\text {base, avg. }}$ The parameter $v_{\text {base, avg }}$ is computed by averaging the time-resolved velocity over $6-8$ cardiac cycles and $v_{\text {peak, avg }}$ is the peak of the temporally averaged velocity via 2 -second sliding window during the course of hyperemia. Computation of $\Delta Q_{\max }$ ignores the very small change in lumen area that is not detectable at the resolution of $1 \mathrm{~mm}$. This leads to an error no greater than $5 \%$ based on previous observations $[16,32]$ where the lumen diameter was found to increase by $3-5 \%$ during peak hyperemia.

\section{Results}

Representative magnitude and phase difference images of the femoral artery and vein are shown in Figure 2. The phase difference image (Figure 2b) demonstrates the susceptibility-induced phase contrast between vein and artery. Figure 2c more clearly shows that the relative difference in the magnetic susceptibility is negligible between tissue and fully oxygenated arterial blood. The temporal position of $\mathrm{HbO}_{2}$ time-course in femoral vein (Figure $2 \mathrm{~d}$ ) is defined with respect to the acquisition time of the center of k-space, e.g. $2.5 \mathrm{~s}, 7.5 \mathrm{~s}, 12.5 \mathrm{~s}$ etc. According to error propagation (eqn. 1) the predominant contributor to standard error of $\mathrm{HbO}_{2}$ is the error in $\Delta \varphi$; conservatively estimated at $\pm 3 \% \mathrm{HbO}_{2}$. During baseline the blood oxygen saturation level is approximately $67 \%$ (dashed horizontal line). In healthy subjects, the observed blood oxygenation at the imaging slice typically ranges from 30 to $85 \%$ post occlusion, representing the saturation level of washed-out oxygendepleted blood and maximum above-baseline value during peak hyperemia, respectively. The range of $\mathrm{HbO}_{2}$ values qualitatively agrees with tissue $\mathrm{HbO}_{2}$ measurements made with near-infrared spectroscopy [12]. Figure 3 shows time-resolved velocity data derived from projection images of the femoral artery. Figure 3a displays time series of projection images, Figure $3 \mathrm{~b}$ the time-course of velocity during hyperemia (expanded in Figure 3c). The latter highlights the sharp increase in the averaged blood flow velocity and monophasic

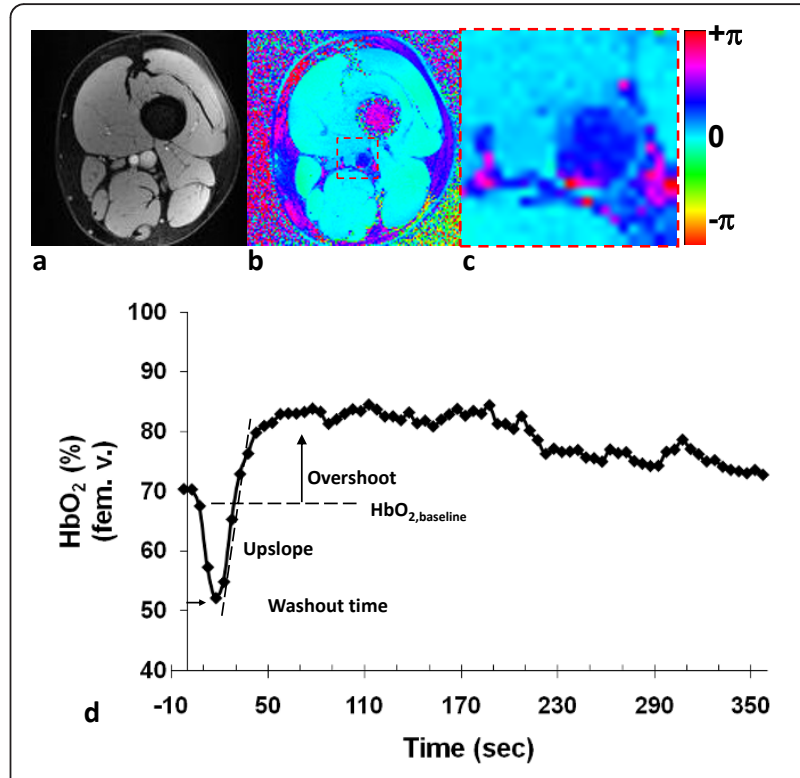

Figure 2 a) Sample magnitude and b) phase difference image for quantifying venous $\mathrm{HbO}_{2}$ in the femoral vein of a healthy young subject. The region in the dashed box is magnified in panel c). d) Representative time-course of $\mathrm{HbO}_{2}$. 


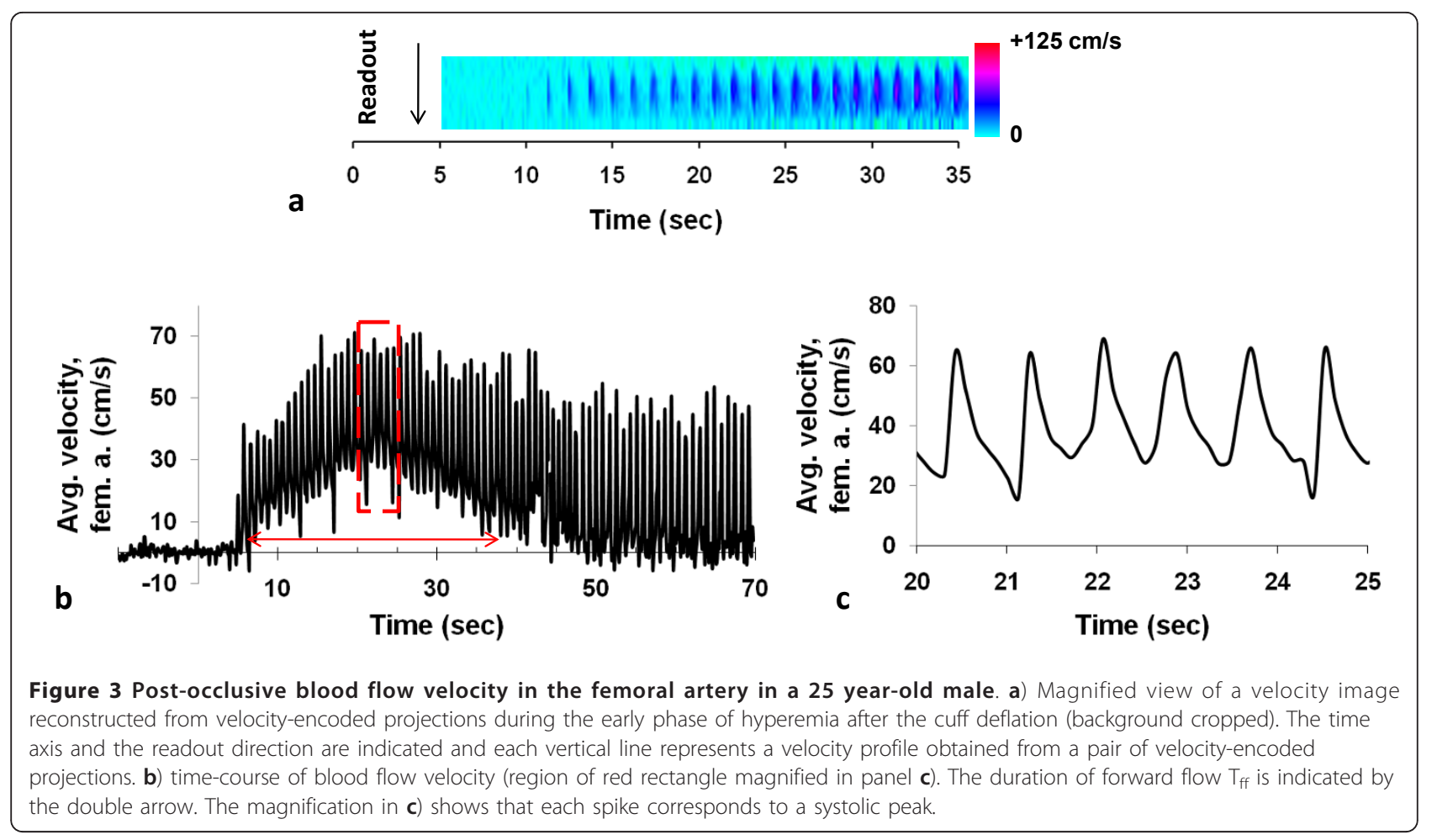

waveform (absence of retrograde flow) during the early phase of hyperemia, presumably due to reduced microvascular resistance. The velocity waveforms as well as the typical peak average velocity agree with previous MR quantifications [15,33]. The results are summarized in Table 1 where the average value and standard deviation (SD) for each parameter are reported for each group. Uncertainty (determined via error propagation) for each parameter is also included under each column heading. Based on a two-tailed t-test, assuming unequal variances, overshoot and TTP were significantly different $(\mathrm{p}<0.05)$ between $\mathrm{YH}$ and $\mathrm{OH}$ subjects. We also observe that the standard deviations of the parameters (all but the overshoot) are greater among the $\mathrm{OH}$, which may reflect longer exposure to different types of lifestyles.

Table 1 Summary of physiological parameters derived from dynamic oximetry and velocimetry.

\begin{tabular}{|c|c|c|c|c|c|c|}
\hline $\begin{array}{l}\text { Age } \\
\text { (yrs) }\end{array}$ & $\begin{array}{l}\text { Washout } \\
( \pm 2.5 \mathrm{sec})\end{array}$ & $\begin{array}{c}\text { Upslope } \\
\left( \pm 0.2 \% \mathrm{HbO}_{2} / \mathrm{sec}\right)\end{array}$ & $\begin{array}{c}\text { Overshoot } \\
\left( \pm 3 \% \mathrm{HbO}_{2}\right)\end{array}$ & $\begin{array}{l}\Delta Q_{\max } \\
\pm 0.5 \\
\end{array}$ & $\begin{array}{c}T_{\mathrm{ff}} \\
( \pm 1 \mathrm{sec}) \\
\end{array}$ & $\begin{array}{c}\text { TTP } \\
( \pm 1 \mathrm{sec}) \\
\end{array}$ \\
\hline \multicolumn{7}{|c|}{ Old $(59 \pm 7.3 y r s)$} \\
\hline 58 & 27.5 & 0.49 & 2 & 3.8 & 28 & 20 \\
\hline 55 & 22.5 & 0.78 & 7 & 10.4 & 25 & 16 \\
\hline 56 & 12.5 & 1.9 & 7 & 8.3 & 30 & 16 \\
\hline 55 & 17.5 & 0.9 & 10 & 13.8 & 15 & 14 \\
\hline 72 & 17.5 & 1.3 & 10 & 5.9 & 26 & 17 \\
\hline $59.2 \pm 7.3$ & $19.5 \pm 5.7$ & $1.07 \pm 0.55$ & $7.2 \pm 3.3$ & $8.44 \pm 3.9$ & $24.8 \pm 5.8$ & $16.6 \pm 2.2$ \\
\hline \multicolumn{7}{|c|}{ Young ( $26 \pm 1.6$ yrs $)$} \\
\hline 25 & 17.5 & 1.2 & 13 & 9.2 & 22 & 14 \\
\hline 26 & 17.5 & 1.8 & 25 & 12 & 25 & 14 \\
\hline 26 & 12.5 & 1.8 & 30 & 12.5 & 30 & 11 \\
\hline 25 & 12.5 & 1.9 & 25 & 7.7 & 20 & 11 \\
\hline 29 & 12.5 & 1.9 & 20 & 6.65 & 25 & 14 \\
\hline $26.2 \pm 1.6$ & $14.5 \pm 2.7$ & $1.72 \pm 0.29$ & $22.6 \pm 6.4$ & $9.61 \pm 2.6$ & $24.4 \pm 3.8$ & $12.8 \pm 1.64$ \\
\hline *p-value & 0.127 & 0.058 & 0.003 & 0.592 & 0.9 & 0.017 \\
\hline
\end{tabular}

*Two-tailed t-test assuming unequal variances. 


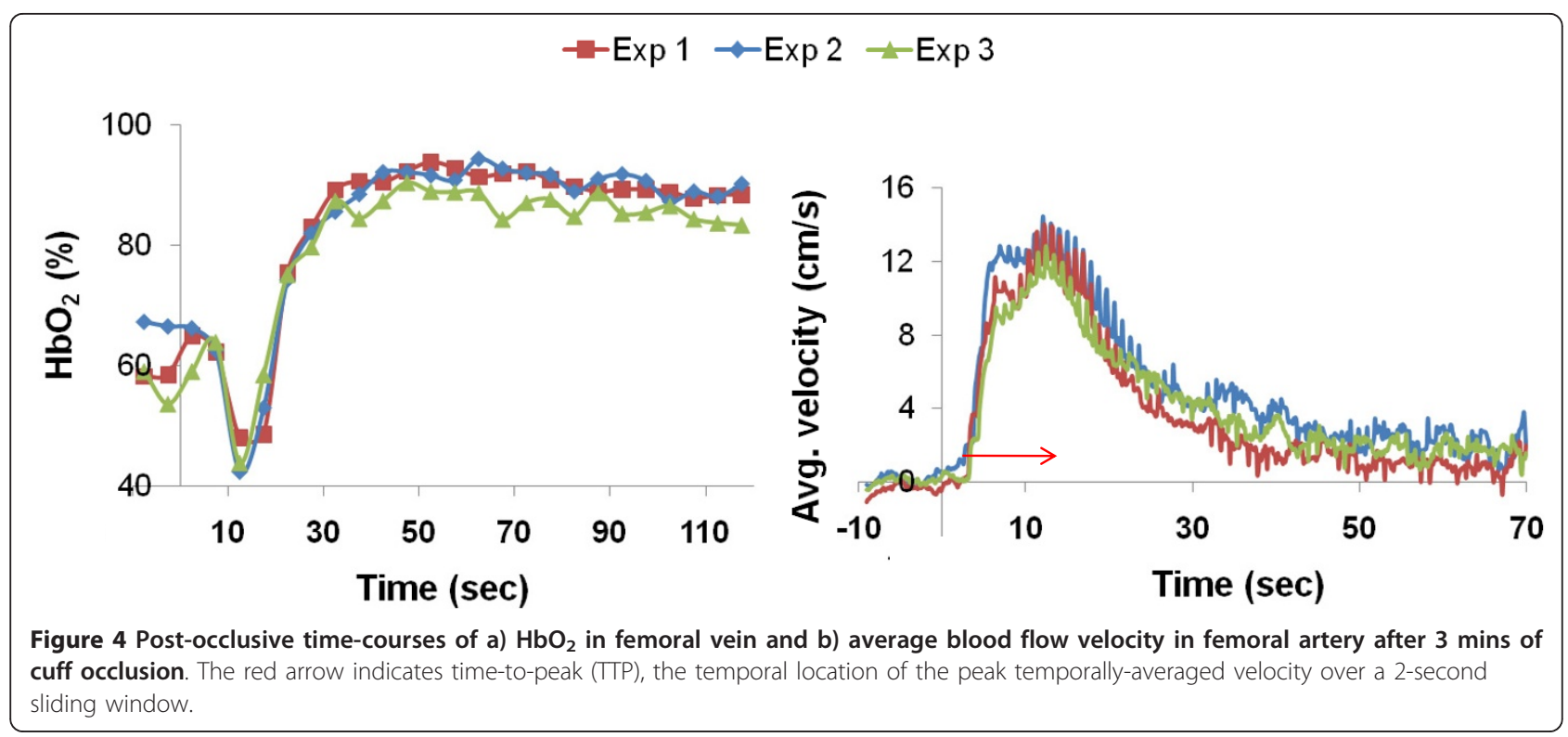

Repeated measurements of $\mathrm{HbO}_{2}$ (Figure 4a) and blood flow velocity (Figure $4 \mathrm{~b}$ ) are shown for one of the subjects. The largest variations between scans in the $\mathrm{HbO}_{2}$ time-course typically occur just prior to cuff release due to the absence of flow-related enhancement. However, this is largely irrelevant since none of the parameters of interest are affected by the pre-cuff-release signal course. In Figure $4 \mathrm{~b}$ the blood flow velocity was averaged over 2 s sliding-window intervals for comparing the velocity time-courses of three measurements. The average oximetric and velocimetric parameters from the three successive scans along with their SD and coefficients of variation $(\mathrm{CV})$ are summarized in Table 2 . For the reproducibility study, the average blood flow velocity at rest

Table 2 Repeat measurements of reactive hyperemia in two healthy subjects.

\begin{tabular}{ccccccc}
\hline & $\begin{array}{c}\text { Washout } \\
(\mathbf{s e c})\end{array}$ & $\begin{array}{c}\text { Upslope } \\
\left(\mathbf{H b O}_{\mathbf{2}} / \mathbf{s e c}\right)\end{array}$ & $\begin{array}{c}\text { Overshoot } \\
\left(\mathbf{H b O}_{2}\right)\end{array}$ & $\begin{array}{c}\mathbf{T}_{\text {ff }} \\
(\mathbf{s e c})\end{array}$ & $\begin{array}{c}\text { TTP } \\
(\mathbf{s e c})\end{array}$ & $\begin{array}{c}\mathbf{v}_{\text {peak, avg }} \\
(\mathbf{c m} / \mathbf{s})\end{array}$ \\
\hline Healthy & $\mathbf{3 9}$ yr-old \\
Exp 1 & 15 & 2 & 30 & 19 & 10 & 13.5 \\
Exp 2 & 12.5 & 1.9 & 32 & 15 & 9 & 13.6 \\
Exp 3 & 12.5 & 2.2 & 30 & 17 & 9 & 12.5 \\
Avg & 13.3 & 2.0 & 30.7 & 17 & 9.3 & 13.2 \\
Std & 1.4 & 0.15 & 1.15 & 2 & 0.58 & 0.61 \\
CV (\%) & 10.5 & 7.5 & 3.7 & 11.8 & 6.2 & 4.6 \\
& & Healthy $\mathbf{2 6}$ yr-old & & & \\
Exp 1 & 22.5 & 1.32 & 11 & 10 & 4.1 & 20.6 \\
Exp 2 & 22.5 & 1.29 & 8 & 12 & 4.4 & 24.6 \\
Exp 3 & 22.5 & 1.54 & 10 & 12 & 4 & 25.4 \\
Avg & 22.5 & 1.38 & 9.7 & 11.3 & 4.17 & 23.5 \\
Std & 0 & 0.136 & 1.53 & 1.15 & 0.21 & 2.57 \\
CV (\%) & 0 & 9.9 & 15.8 & 10.2 & 5.0 & 10.9 \\
\hline
\end{tabular}

was not quantified (therefore $\Delta \mathrm{Q}_{\max }$ could not be computed). Instead, we report peak average velocity, $\mathrm{v}_{\text {peak, avg, }}$ (Figure $4 \mathrm{~b}$ ). For both subjects the average CV is approximately $8 \%$ but the $\mathrm{CV}$ was as high as $16 \%$ (overshoot in 26 yr-old subject). However, it should be noted that the intersubject SD is about 50\% and thus much greater than intrasubject SD (Table 1) for all parameters.

\section{Discussion}

The work shows that simultaneous measurement of dynamic oximetric and arterial flow velocity is possible for assessing post-occlusive peripheral vascular reactivity. In contrast, a sequential measurements would require application of the cuff twice, which would not be tolerable by a significant number of patients. The method returns six parameters, three each related to the time-course of venous $\mathrm{HbO} 2$, and arterial flow velocity. The time-course of $\mathrm{HbO}_{2}$ (referred to as dynamic oximetry) follows the fate of the oxygen-depleted blood in the capillary bed upon restoration of flow and its subsequent replacement by normally oxygenated venous blood. It thus provides quantitative information on how rapidly oxygen-depleted blood is washed out of tissue as well as the rate at which tissue is resaturated. In addition, the overshoot may reflect the magnitude of hyperemia since tissue has reduced time to extract $\mathrm{O}_{2}$, a timeconsuming process driven by perfusion, thus greater above-baseline $\mathrm{HbO}_{2}$ is expected with more pronounced hyperemia. The oximetric parameters derived in this pilot study are consistent with those of the authors' prior work in which a significantly greater overshoot was observed in healthy young compared to older subjects [18]. 
The velocity profile of the healthy subjects qualitatively agrees with previous "real-time" phase-contrast CMR data $[15,16]$ including the duration of forward flow $\left(\mathrm{T}_{\mathrm{FF}} ; 20-35 \mathrm{~s}\right)$ and peak average velocity $(\sim 40-$ $60 \mathrm{~cm} / \mathrm{s})$. The unique feature of the "real-time" techniques $[15,16]$, including the current method, is the ability to resolve the velocity waveform for each cardiac cycle during hyperemia. In all cases, the blood flow direction does not reverse during the early phase of hyperemia (Figure 3b) due to significant reduction in microvascular resistance. Since vasodilation is generally triggered by NO release, TTP and $\mathrm{T}_{\mathrm{ff}}$ may assess the microvascular function's response to an ischemia, i.e. the rate and maximum bioavailability of nitric oxide release.

The projection method has the usual limitation when a spatial dimension is sacrificed for improved temporal resolution in that velocity is resolved along a single spatial direction only and thus inherently averaged along the projected lumen. Hence, peak velocity cannot be quantified but rather an average in the projection direction is extracted. Lastly, "misregistration" of femoral vessels between reference image and projections during hyperemia is largely avoided by applying the cuff 10 to $15 \mathrm{~cm}$ from the imaging slice, and since the blood velocity near the vessel wall is nearly zero the error in average velocity is expected to be small.

The proposed method is a component of an ongoing development of CMR-based techniques for measuring multiple parameters across different vascular territories as part of a single examination to study endothelial dysfunction.

\section{Conclusions}

The quantitative imaging method introduced provides a series of parameters on peripheral vascular reactivity in about fifteen minutes, allowing time for additional quantification of physiological parameters in a single scan session and should be of value to study preclinical cardiovascular disease.

\section{Acknowledgements \\ The work was supported by the grants NIH R21 HL088182, NIH RC1- HL099861 and R01 HL109545.}

\section{Authors' contributions}

ML conceived, designed and implemented the pulse sequence, collected and analyzed the data. FW participated in the design of experiment and study, and helped to draft the manuscript. All authors read and approved the final manuscript.

\section{Competing interests}

The authors declare that they have no competing interests.

Received: 11 July 2011 Accepted: 28 October 2011

Published: 28 October 2011

\section{References}

1. Lloyd-Jones D, Adams RJ, Brown TM, Carnethon M, Dai S, De Simone G, Ferguson TB, Ford E, Furie K, Gillespie C, et al: Heart disease and stroke statistics-2010 update: a report from the American Heart Association. Circulation 2010, 121:e46-e215.

2. Ogden CL, Carroll MD, Curtin LR, McDowell MA, Tabak CJ, Flegal KM: Prevalence of overweight and obesity in the United States, 1999-2004. Jama 2006, 295:1549-1555.

3. Ogden $C L$, Carroll MD, McDowell MA, Flegal KM: Obesity among adults in the United States-no statistically significant chance since 2003-2004. NCHS data brief 2007, 1-8.

4. National Center for Health Statistics: Health, United States, 2007. With chartbook on trends in the health of Americans Hyattsville, MD: National Center for Health Statistics; 2007.

5. Centers for Disease Control and Prevention: Prevalence of self-reported physically active adults-United States, 2007. MMWR 2008;57:1297-1300. Book Centers for Disease Control and Prevention. Prevalence of self-reported physically active adults-United States, 2007. MMWR 2008, 57:1297-1300.

6. Centers for Disease Control and Prevention: Youth risk behavior surveillance-United States, 2007. MMWR 2008, 57(SS-04):1-131.

7. BRFSS prevalence and trends data [Internet]. Atlanta, GA: Centers for Disease Control and Prevention; 2008 [http://apps.nccd.cdc.gov/brfss/page. asp?cat=AC\&yr=2007\&state $=$ US\#AC]

8. Ludmer PL, Selwyn AP, Shook TL, Wayne RR, Mudge GH, Alexander RW, Ganz P: Paradoxical vasoconstriction induced by acetylcholine in atherosclerotic coronary arteries. N Engl J Med 1986, 315:1046-1051.

9. Widlansky ME, Gokce N, Keaney JF Jr, Vita JA: The clinical implications of endothelial dysfunction. Journal of the American College of Cardiology 2003, 42:1149-1160.

10. Boushel R, Piantadosi CA: Near-infrared spectroscopy for monitoring muscle oxygenation. Acta Physiol Scand 2000, 168:615-622.

11. Kragelj R, Jarm T, Erjavec T, Presern-Strukelj M, Miklavcic D: Parameters of postocclusive reactive hyperemia measured by near infrared spectroscopy in patients with peripheral vascular disease and in healthy volunteers. Annals of biomedical engineering 2001, 29:311-320.

12. Yu G, Durduran T, Lech G, Zhou C, Chance B, Mohler ER, Yodh AG: Timedependent blood flow and oxygenation in human skeletal muscles measured with noninvasive near-infrared diffuse optical spectroscopies. J Biomed Opt 2005, 10:024027.

13. Franceschini $M$, Fantini $S$, Palumbo $R$, Pasqualini $L$, Vaudo $G$, Franceschini $E$, Gratton E, Palumbo B, Innocente S, Mannarino E: Quantitative nearinfrared spectroscopy on patients with peripheral vascular disease. In Proc SPIE; San Diego. Edited by: Benaron DA, Chance B, Ferrari M. SPIE; 1997:112-115.

14. Dupuis J, Arsenault A, Meloche B, Harel F, Staniloae C, Gregoire J: Quantitative hyperemic reactivity in opposed limbs during myocardial perfusion imaging: a new marker of coronary artery disease. Journal of the American College of Cardiology 2004, 44:1473-1477.

15. Mohiaddin RH, Gatehouse D, Moon JC, Youssuffidin M, Yang GZ, Firmin DN, Pennell DJ: Assessment of reactive hyperaemia using real time zonal echo-planar flow imaging. J Cardiovasc Magn Reson 2002, 4:283-287.

16. Schwitter J, Oelhafen M, Wyss BM, Kozerke S, Amann-Vesti B, Luscher TF, Boesiger P: 2D-spatially-selective real-time magnetic resonance imaging for the assessment of microvascular function and its relation to the cardiovascular risk profile. J Cardiovasc Magn Reson 2006, 8:759-769.

17. Wu WC, Mohler E, Ratcliffe SJ, Wehrli FW, Detre JA, Floyd TF: Skeletal muscle microvascular flow in progressive peripheral artery disease: assessment with continuous arterial spin-labeling perfusion magnetic resonance imaging. J Am Coll Cardiol 2009, 53:2372-2377.

18. Langham MC, Floyd TF, Mohler ER, Magland JF, Wehrli FW: Evaluation of cuff-induced ischemia in the lower extremity by magnetic resonance oximetry. J Am Coll Cardiol 2010, 55:598-606.

19. Corretti MC, Anderson TJ, Benjamin EJ, Celermajer D, Charbonneau F, Creager MA, Deanfield J, Drexler H, Gerhard-Herman M, Herrington D, et al: Guidelines for the ultrasound assessment of endothelial-dependent flow-mediated vasodilation of the brachial artery: a report of the International Brachial Artery Reactivity Task Force. Journal of the American College of Cardiology 2002, 39:257-265.

20. Kurth CD, Steven JM, Benaron D, Chance B: Near-infrared monitoring of the cerebral circulation. J Clin Monit 1993, 9:163-170. 
21. Celermajer DS, Sorensen KE, Gooch VM, Spiegelhalter DJ, Miller OI, Sullivan ID, Lloyd JK, Deanfield JE: Non-invasive detection of endothelial dysfunction in children and adults at risk of atherosclerosis. Lancet 1992, 340:1111-1115.

22. Karatzis EN, Ikonomidis I, Vamvakou GD, Papaioannou TG, Protogerou AD, Andreadou I, Voidonikola PT, Karatzi KN, Papamichael CM, Lekakis JP: Longterm prognostic role of flow-mediated dilatation of the brachial artery after acute coronary syndromes without ST elevation. The American journal of cardiology 2006, 98:1424-1428.

23. Katz SD, Hryniewicz K, Hriljac I, Balidemaj K, Dimayuga C, Hudaihed A, Yasskiy A: Vascular endothelial dysfunction and mortality risk in patients with chronic heart failure. Circulation 2005, 111:310-314

24. De Roos NM, Bots ML, Schouten EG, Katan MB: Within-subject variability of flow-mediated vasodilation of the brachial artery in healthy men and women: implications for experimental studies. Ultrasound in medicine \& biology 2003, 29:401-406.

25. Potter K, Reed CJ, Green DJ, Hankey GJ, Arnolda LF: Ultrasound settings significantly alter arterial lumen and wall thickness measurements. Cardiovasc Ultrasound 2008, 6:6.

26. Benjamin EJ, Larson MG, Keyes MJ, Mitchell GF, Vasan RS, Keaney JF Jr, Lehman BT, Fan S, Osypiuk E, Vita JA: Clinical correlates and heritability of flow-mediated dilation in the community: the Framingham Heart Study. Circulation 2004, 109:613-619.

27. Haacke EM, Lai S, Reichenbach JR, Kuppusamy K, Hoogenraad FGC, Takeichi H, Lin W: In Vivo Measurement of Blood Oxygen Saturation Using Magnetic Resonance Imaging: A Direct Validation of the Blood Oxygen Level-Dependent Concept in Functional Brain Imaging. Human Brain Mapping 1997, 5:341-346.

28. Fernández-Seara M, Detre JA, Techawiboonwong A, Wehrli FW: MR susceptometry for measuring global brain oxygen extraction. Magn Reson Med 2006, 55:967-973.

29. Langham MC, Jain V, Magland JF, Wehrli FW: Time-resolved absolute velocity quantification with projections. Magn Reson Med 2010, 64:1599-1606

30. Langham MC, Magland JF, Floyd TF, Wehrli FW: Retrospective correction for induced magnetic field inhomogeneity in measurements of largevessel hemoglobin oxygen saturation by MR susceptometry. Magn Reson Med 2009, 61:626-633.

31. Spees WM, Yablonskiy DA, Oswood MC, Ackerman JJ: Water proton MR properties of human blood at 1.5 Tesla: magnetic susceptibility, $T(1), T$ (2), $T^{*}(2)$, and non-Lorentzian signal behavior. Magn Reson Med 2001, 45:533-542.

32. Silber HA, Ouyang P, Bluemke DA, Gupta SN, Foo TK, Lima JA: Why is flowmediated dilation dependent on arterial size? Assessment of the shear stimulus using phase-contrast magnetic resonance imaging. Am J Physiol Heart Circ Physiol 2005, 288:H822-828.

33. Oelhafen M, Schwitter J, Kozerke S, Luechinger R, Boesiger P: Assessing arterial blood flow and vessel area variations using real-time zonal phase-contrast MRI. J Magn Reson Imaging 2006, 23:422-429.

doi:10.1186/1532-429X-13-66

Cite this article as: Langham and Wehrli: Simultaneous mapping of temporally-resolved blood flow velocity and oxygenation in femoral artery and vein during reactive hyperemia. Journal of Cardiovascular Magnetic Resonance 2011 13:66.

\section{Submit your next manuscript to BioMed Central and take full advantage of:}

- Convenient online submission

- Thorough peer review

- No space constraints or color figure charges

- Immediate publication on acceptance

- Inclusion in PubMed, CAS, Scopus and Google Scholar

- Research which is freely available for redistribution

Submit your manuscript at www.biomedcentral.com/submit
Biomed Central 